\title{
Shape and Scale Dependent Diffusivity of Colloidal
}

\section{Nanoclusters and Aggregates}

\author{
Maria Michiko T. Alcanzare, ${ }^{\dagger}$ Santtu T. T. Ollila, ${ }^{\dagger}$ Vaibhav Thakore, ${ }^{*, \dagger}$ Aleena Maria

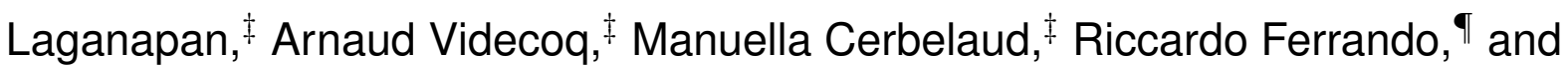 \\ Tapio Ala-Nissila ${ }^{\dagger, \S}$ \\ COMP CoE at the Department of Applied Physics, Aalto University School of Science, \\ FIN-00076 Aalto, Espoo, Finland, SPCTS, UMR 7315, ENSCI, CNRS, Centre Européen \\ de la Céramique, 12 rue Atlantis, 87068 Limoges, France, Dipartimento di Fisica, \\ Università degli Studi di Genova, Via Dodecaneso, 33, 16146 Genova, Italy, and \\ Department of Physics, Brown University, Providence, Rhode Island 02912-1843, USA \\ E-mail: vaibhav.thakore@aalto.fi
}

KEYWORDS: nanoscale colloidal diffusion, fluctuating lattice Boltzmann method, shape and size dependent diffusivity, Stokes-Einstein diffusion, Brownian limit, no-slip and full-slip microscopic boundary conditions, hydration layer volume fraction

\footnotetext{
${ }^{*}$ To whom correspondence should be addressed

${ }^{\dagger}$ Aalto University

${ }^{\ddagger}$ Science des Procédés Céramique et de Traitements de Surface

I University of Genoa

$\S$ Brown University
} 


\begin{abstract}
Diffusion of colloidal nanoparticles and nanomolecular aggregates plays an important role in various biophysical and physicochemical phenomena of interest in several engineering applications. While the diffusive transport of submicron colloidal particles is well characterized, the diffusive behavior of nanoscale colloids is not well understood. Here, we present results for the shape and size dependent diffusion of colloidal nanoparticles, fused nanoclusters and nanoaggregates using a fluctuating lattice Boltzmann method. We use physically realistic parameters characteristic of an aqueous solution, with explicitly implemented microscopic no-slip and full-slip velocity boundary conditions at the colloid-fluid interface. Results from nanocolloids of various sizes below $10 \mathrm{~nm}$ demonstrate how increased volume fraction of the hydration layer significantly influences diffusivities. Full-slip colloids are found to diffuse faster than no-slip particles. We also characterize the shape dependent anisotropy of the diffusion coefficients of nanoclusters through the Green-Kubo relation. Finally, we also study the size dependence of the diffusion of nanoaggregates comprising $N \leq 108$ monomers and demonstrate that the diffusion coefficient approaches the expected continuum scaling limit of $N^{-1 / 3}$.
\end{abstract}

Understanding the dynamics and transport properties of colloidal nanoparticles and nanomolecular aggregates is crucially important for several applications in chemical, mechanical, materials and biomedical engineering. Important examples include the possible enhancement of heat transfer in nanofluids, 14 the use of functionalized nanoparticles or bioengineered macromolecules for targeted drug and genetic material delivery in therapeutics, micro or nanofluidics based biomolecular sensor devices, $\frac{[56}{\sqrt{6}}$ nanoscale self-assembly of colloidal crystals, ${ }^{7}$ ceramic processing,,$[9]$ and coatings. Colloidal dynamics in slowly evolving systems, such as biological fluids or suspensions under quasi-equilbrium conditions, are characterized by diffusive transport in the presence of solvent mediated hydrodynamic interactions. The diffusive behavior of submicron $(100-1000 \mathrm{~nm})$ colloidal particles in such systems has been extensively studied theoretically, and is now well understood also exper- 
imentally due to recent advances in the microscale self-assembly of spherical colloids. 10,12 However, the most interesting size or scale dependent physicochemical phenomena become emergent at much smaller length scales. Recent advances in size-selective controlled synthesis have made nanoscale $(1-10 \mathrm{~nm})$ particles commercially available, but their precise characterization in solvents remains complicated due to their small size.

The fundamental quantity that characterizes colloidal dynamics is the Brownian diffusion coefficient $D$, which follows the canonical Stokes-Einstein (SE) relation,

$$
D=\frac{k_{B} T}{\gamma 6 \pi \eta R}
$$

for a spherical particle of radius $R$. Here $k_{B} T$ and $\eta$ are the thermal energy and viscosity of the fluid, respectively. The SE relation has also been extended to non-spherical particles with shapes corresponding to regular geometric objects, such as oblate and prolate spheroids, and cylinders.13 The Brownian limit of colloidal diffusion, applicable to regimes where the SE relation is valid, assumes that a colloid is massive and slow as compared to the surrounding solvent particles. ${ }^{14-16}$ Additionally, it further assumes that the colloids undergo uncorrelated random motion in a quiescent fluid continuum as described by the Stokes equation. However, while the single particle Brownian dynamics is well characterized, the simulation of the many-particle dynamics of dense Brownian supensions remains a challenge due to manybody hydrodynamic interaction and correlation effects. .17

The simple SE relation in eq (1) contains a subtlety related to the prefactor $\gamma$ in the denominator, which takes values of unity and $2 / 3$ corresponding to no-slip and full-slip velocity boundary conditions at the colloid-fluid interface, respectively. The no-slip boundary condition is typically considered to arise as a consequence of the roughness resulting from molecular scale irregularities on the surface of a colloid. $\frac{[1819}{19}$ The use of the no-slip boundary condition, however, has no firm theoretical basis and its use over the years has been justified on the basis of experiments with colloids in the Brownian limit. ${ }^{19 \mid 22]}$ However, recent 


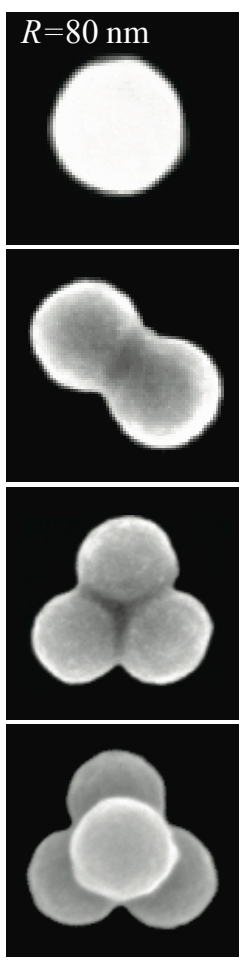

A

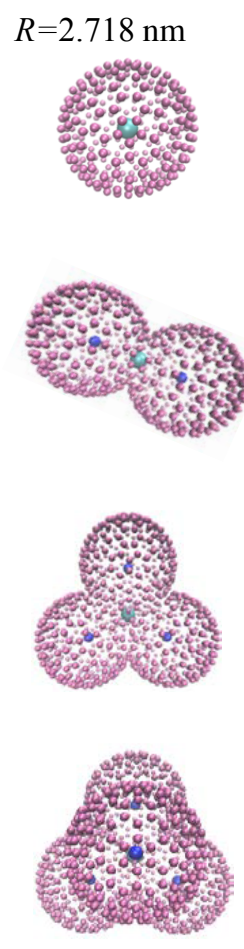

B
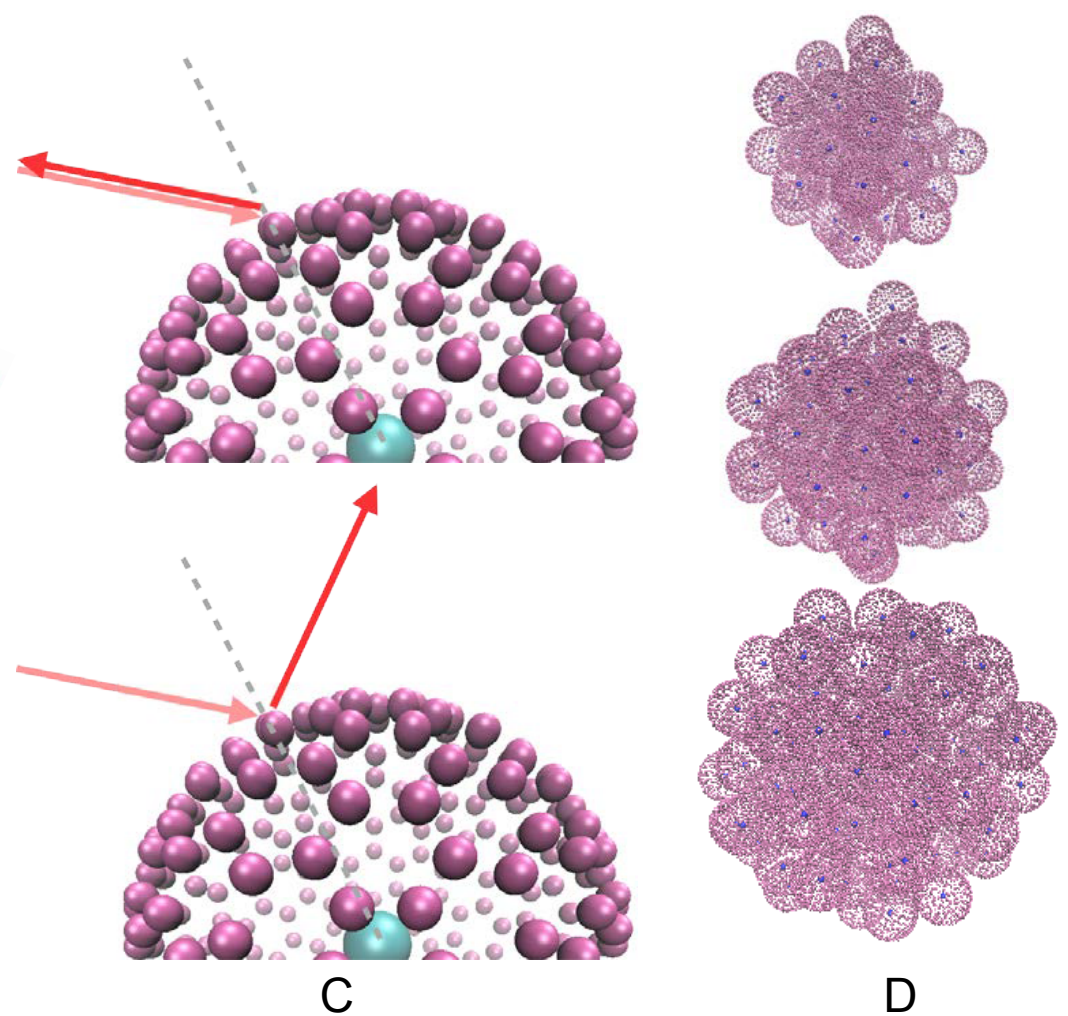

D

Figure 1: (A) Fused submicron colloids synthesized using $80 \mathrm{~nm}$ polystyrene particles ${ }^{12 *}$. (B-D) A graphical representation of the colloidal nanoparticles and aggregates used in the FLB simulations. (B) Spherical monomers represented by nodes placed at the vertices of a 320-icosahedron, dimer, trimer and tetramer comprising two, three and four spherical monomers, respectively. (C) Implementation of the no-slip (top) and the full-slip (bottom) velocity boundary conditions with arrows representing the collisions between a surface node of the colloid and a fluid volume element. The no-slip boundary condition is set such that the initial relative velocity of the colloid surface node and the fluid volume element reverses its direction after a collision. The full-slip boundary condition is set by forcing the initial and final tangential components of the relative velocity of the colloid surface node and the fluid volume element to be equal. (D) (top-bottom) Colloidal aggregates with 55, 80 and 101 non-overlapping spherical monomers.

*Reproduced from "3D Brownian Diffusion of Submicron-Sized Particle Clusters", Martin Hoffmann, Claudia S. Wagner, Ludger Harnau, and Alexander Wittemann ACS Nano 2009 3 (10), 3326-3334. Copyright (2009) American Chemical Society.

experimental studies involving diffusion of mesoscale colloids have revealed a more complex dependence of the velocity boundary condition on the nature of the solute-solvent physicochemical interactions, that gives rise to boundary conditions ranging from no-slip to partial 
and full-slip. $20123 \sqrt[27]{27}$ The strength and the nature of the interactions between the colloid surface and the solvent molecules not only influence the velocity boundary condition at the colloid-fluid interface, but also simultaneously determine the thickness of the hydration layer associated with the surface of the colloid. The dynamics of the solvent molecules within hydration layers associated with the surfaces of colloidal macromolecules and the thickness of the hydration layer have been a subject of intense investigation and debate recently, as a result of advances in terahertz and NMR spectroscopies. $\frac{28}{32}$ However, the role of hydration layer in the diffusion of nanoscale colloids, wherein the volume of the colloidal nanoparticle becomes comparable to the volume of the hydration layer associated with its surface, has not been considered.

In principle, it is possible to model colloidal dynamics by using full-scale molecular dynamics (MD) simulations that take into account all possible interactions between solvent molecules and colloidal particles. However, this turns out to be computationally prohibitively expensive for the calculation of the Brownian transport coefficients because of the disparate time and length scales involved in solvent relaxation and colloidal motion mediated through many-body hydrodynamic interactions. $\frac{\sqrt{33}}{}$ This has led to the development of hybrid methods such as Brownian dynamics (BD) that incorporates hydrodynamic interactions using either the analytic Oseen or the Yamakawa-Rotne-Prager (YRP) tensor in the Stokes limit, $\underline{34}$ and mesoscopic methods that model fluid dynamics using stochastic coarse-grained hydrodynamics. $\frac{35}{3}$ Unlike BD, the stochastic coarse-grained hydrodynamics solvers are designed to recover the full Navier-Stokes equations in the continuum limit while conserving mass and momentum locally. Such recently developed mesoscopic methods coupled to MD simulation

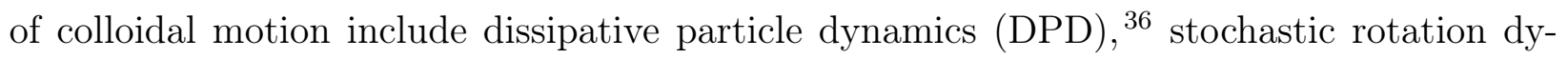
namics (SRD), $\underline{35137}$ and the fluctuating lattice Boltzmann (FLB) method. $\underline{38139}$ A summary of the relative advantages and disadvantages of these methods in the context of simulating colloidal dynamics and transport phenomena can be found in a recent article by Tomilov et al. $\underline{40}$ 
Our main focus in this work is the shape and size dependent Brownian diffusivity of fused colloidal nanoclusters and nanoaggregates in a solvent modeled with physically relevant parameters corresponding to an aqueous solution at room temperature. To this end, we employ the hybrid fluctuating lattice Boltzmann (FLB) - molecular dynamics (MD) method

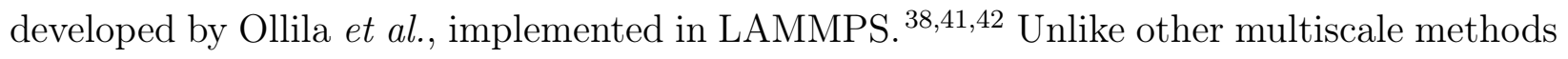
discussed above, the FLB method uses spatially extended particles and explicitly implemented microscopic no-slip and full-slip velocity boundary conditions at the colloid-fluid interface. The method incorporates full non-linear hydrodynamic interactions between the colloidal particles and a realistic coupling of the thermal fluctuations to the particles through a fluctuating solvent in accordance with the Brownian limit.

Using FLB, we study the diffusive behavior of fused colloidal nanoclusters (spheres, dimers, trimers and tetramers) to understand the role of the hydration layer in the shape and size dependent diffusivity of colloids; the differences in their diffusivities corresponding to no-slip and full-slip velocity boundary conditions; the effect of the variations in the degree of overlap of the spherical monomer constituents on the diffusivity of the fused nanoclusters; and, the anisotropic diffusivity along their axes of symmetry. Additionally, we also investigate the size-dependent scaling of the diffusivities of the larger nanocluster aggregates as a function of the increase in the number $N$ of the spherical nanoparticles comprising them.

The colloidal particles in the $1-10 \mathrm{~nm}$ range are represented by using evenly distributed nodes on their surface (see Figure 1 B-D) with an approximate area of $0.29 \mathrm{~nm}^{2}$ per node equivalent to an internode spacing of $0.4 \Delta x$ on the colloid surface, smaller than the prescribed

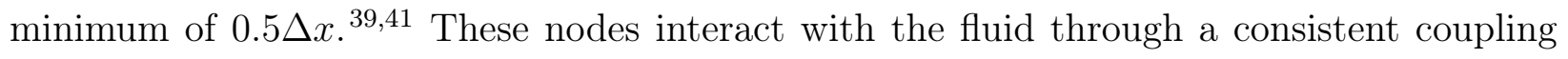
scheme based on a conservative interaction. ${ }^{41}$ The node masses are fixed such that the mass of the colloid equals twice the mass of the volume of the fluid displaced by the colloid. The density and viscosity of the fluid are set to $9.98 \times 10^{2} \mathrm{~kg} \mathrm{~m}^{-3}$ and $1.0 \times 10^{-3} \mathrm{Pas}$, respectively. The simulation domain is discretized using a lattice spacing of $1.2 \mathrm{~nm}$ and a time step of $2.25 \times 10^{-4} \mathrm{~ns}$ is employed in all simulations. 
Single particle diffusion is simulated by placing the colloid in a cubic box with periodic boundaries and a side of length $L$. The mean square displacement (MSD) of the colloidal particle is tracked and the relation

$$
D=\lim _{\Delta t \rightarrow \infty} \frac{\left\langle|\vec{r}(t+\Delta t)-\vec{r}(t)|^{2}\right\rangle}{6 \Delta t},
$$

is used to calculate the diffusion coefficient. In practice, $D$ is obtained by calculating the slope of the MSD when it begins to vary linearly with time. Periodic boundary conditions give rise to finite-size effects due to the hydrodynamic interactions. ${ }^{[4 / 38 / 43}[46]$ They are quantified to first-order using 44

$$
D_{\mathrm{NS}}(L)=\frac{k_{B} T}{6 \pi \eta}\left(\frac{1}{R_{H}}-\frac{B}{L}\right),
$$

for no-slip and

$$
D_{\mathrm{FS}}(L)=\frac{k_{B} T}{4 \pi \eta}\left(\frac{1}{R_{H}}-\frac{2 B}{3 L}\right) .
$$

for full-slip spherical colloids of hydrodynamic radius $R_{H}$. Here $B$ is a dimensionless constant. The SE diffusion coefficient for the colloid is then extrapolated using simulation results from different sizes $L$ of the cubic simulation domains. The hydrodynamic radius of an arbitrarily shaped colloidal nanocluster or aggregate calculated from its diffusion coefficient is characteristic of both its size and shape. Because eqs (3) and (4) depend only on $R_{H}$, we employ them to describe the finite size-effects for colloidal particles of all shapes and sizes. Consistent with the theoretically calculated values for $B$, our simulation results indicated values of $B=2.835 \pm 0.025$ and $B=2.882 \pm 0.045$ for the no-slip and full-slip colloids (spheres, dimers, trimers and tetramers) respectively (See Supporting Information, Figures S1 A and B).

For no-slip colloidal spheres of radii $R=2.718,4.330$ and $7.210 \mathrm{~nm}$, our simulation results show diffusivities of $D=6.43 \times 10^{-2}, 4.35 \times 10^{-2}$ and $2.77 \times 10^{-2} \mathrm{~nm}^{2} / \mathrm{ns}$, respectively. These values were found to be smaller than the corresponding SE diffusivities of $D_{S E}=8.08 \times 10^{-2}$, $5.08 \times 10^{-2}$, and $3.05 \times 10^{-2} \mathrm{~nm}^{2} / \mathrm{ns}$, for the colloids calculated by substituting their bare 
particle radii $R$ in eq (1). Thus the effective hydrodynamic radii of the nanoscale colloids in FLB simulations are larger than their bare physical radii. This increase in the effective size of the nanocolloids indicates the presence of an effective boundary layer of thickness $\delta R$, which we define here simply by $\delta R \equiv R_{H}-R=0.7 \pm 0.02 \mathrm{~nm}$. Our model does not explicitly include any physicochemical interactions between the colloid surface and the solvent. The microscopic no-slip velocity boundary condition implemented at the colloid-fluid interface in FLB results in a solvent boundary layer with zero tangential flux of the fluid at the colloid surface. Within this solvent boundary layer the tangential relative fluid velocity gradually increases from zero to bulk values away from the surface of the colloid. This layer of fluid associated with the colloid surface is observed to originate purely as a consequence of the microscopic no-slip velocity boundary condition implemented at the colloid-fluid interface and is heretofore referred to as a hydration layer. Since the no-slip boundary condition is implemented in a similar fashion for all colloids, we expect $\delta R$ to have the same value for the fused clusters as well.

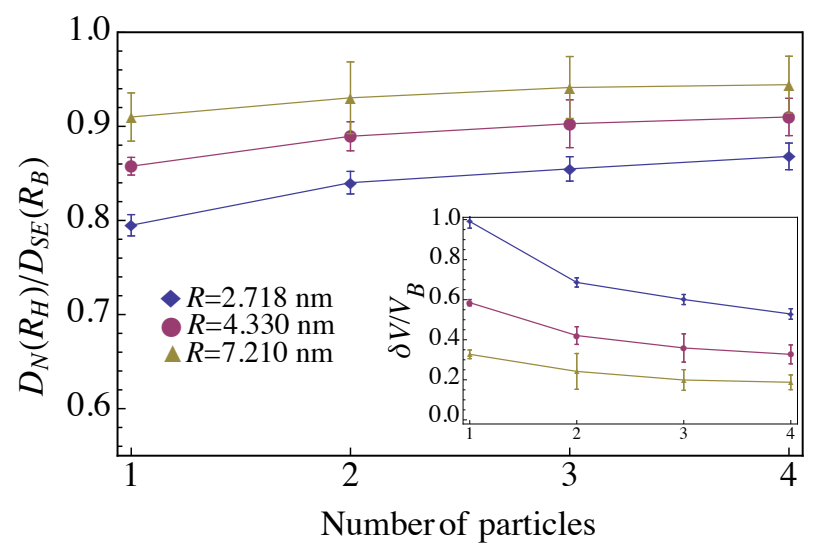

Figure 2: Ratios of the diffusivities of the fused colloidal nanoparticles $D_{N}$ obtained from FLB simulations to their diffusivities $D_{S E}$ calculated from the SE relation using their effective bare radii $R_{B}$. Inset: Variation of the volume fraction of the hydration layer $\delta V / V_{B}$ with change in the number $N$ of the monomers in the fused clusters, where $\delta V=(4 / 3) \pi\left(R_{H}^{3}-R_{B}^{3}\right)$ and $V_{B}=(4 / 3) \pi R_{B}^{3}$.

Figures 2 and 3 illustrate the effect of an increase in the volume fraction of the hydration layer $\delta V / V_{B}$ on the diffusivities of spherical and fused no-slip nanoclusters that are built 
using spherical monomers of radii $R=7.210,4.330$ and $2.718 \mathrm{~nm}$. For fused clusters and aggregates, similar to the monomers, we define an effective bare radius by $R_{B} \equiv R_{H}-\delta R$. An examination of Figure 2 reveals that the ratios of the diffusivities obtained from FLB simulations to those calculated from the SE relation by using the effective bare radii $R_{B}$ tend to approach the Brownian limit of unity with an increase in the size of the constituent spherical monomers in the fused colloidal nanoclusters. The deviations from the Brownian limit in Figure 2 also become more pronounced with a reduction in the number $N$ of the constituent monomer spheres as one moves from colloidal tetramers through trimers and dimers to a spherical nanocolloid monomer of a given size. This can be attributed to an increase in the relative volume fraction of the hydration layer resulting from a decrease in the number $N$ of monomers in the fused nanoclusters (See Figure 2 inset).

Figure 3 further compares the diffusivity data for fused nanoclusters with results from a recent experimental study on diffusivities of fused submicron colloidal clusters that are synthesized with well defined shapes using polystyrene spheres of $80 \mathrm{~nm}$ radii (Figure 1 A). 12 Regardless of the size of the spherical monomer constituents, the normalized diffusivities $D_{N} / D_{1}$ for all fused colloidal clusters are seen to decrease with an increase in the number $N$ of their monomers (Figure 3). However, the normalized diffusivities obtained from the FLB simulations for fused nanocolloids are seen to be systematically greater than those obtained from experiments with the submicron colloids. $\frac{12}{12}$ Moreover, the normalized diffusivites for the fused nanoclusters tend to approach the experimental results with an increase in their monomer size from $R=2.718$ and $4.330 \mathrm{~nm}$ to $7.210 \mathrm{~nm}$. These results again indicate that the diffusion coefficients for the nanoscale colloidal spheres are much smaller than those obtained from the Stokes-Einstein relation using the bare monomer radii $R$. This gives rise to higher values of normalized diffusivities for the fused nanoclusters in contrast to the experimental results from the fused submicron colloids in the Brownian limit.12

Similar to the hydration layer thicknesses on the order of $0.7 \mathrm{~nm}$ observed in our simulations, recent experiments with nanoscale colloids have shown that hydration layers associated 


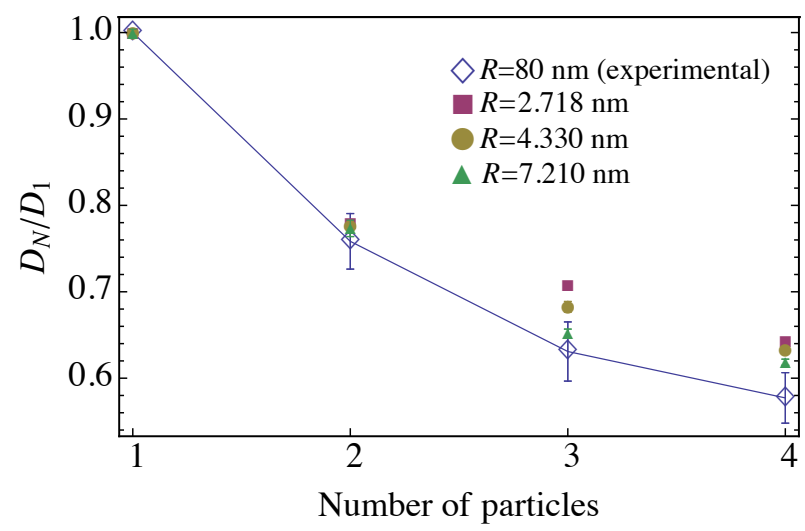

Figure 3: Diffusivities of the fused colloidal nanoclusters comprising $N$ monomers, normalized by the diffusion coefficient of their spherical monomer constituents. The diamonds represent the experimental data for similarly shaped fused colloidal clusters synthesized using $80 \mathrm{~nm}$ spherical polystyrene particles. 12

with colloidal nanoparticles range from $0.3-2.0 \mathrm{~nm}$ in thickness. $\frac{28|30| 47}{}$ Because hydration layer thickness depends on the nature of the physicochemical interaction between the colloid surface and the solvent, it is expected to be largely independent of the colloid size. Also, the hydration layer thickness accounts for a small fraction of the hydrodynamic radius of a large Brownian colloid. Thus, for a Brownian colloid its hydrodynamic radius $R_{H}$ approximately equals its effective bare physical radius $R_{B}$. The contribution of the hydration layer to the diffusivity of the larger colloidal particles in the Stokes-Einstein relation therefore gets masked. However, as we have shown here, for the smaller nanocolloids $(1-10$ $\mathrm{nm}$ in size) the hydration layer contributes significantly to the Stokes-Einstein diffusivity. Although the hydration layer thickness and thus the volume fraction of the hydration layer associated with the fused submicron colloids used in the experimental study by Hofmann et $a l .12$ cannot be inferred directly from their results, a comparison of Figures 2 and 3 clearly highlights the effect of the increased hydration layer volume fraction on colloidal diffusivity at the nanoscale.

Synthesis of fused nanoclusters under experimental conditions invariably leads to a distribution in the degrees of overlap of the spherical monomers fused together to form the nanoclusters. We characterize this by an overlap parameter $\lambda \equiv 1-d /(2 R)$, where $d$ is the 


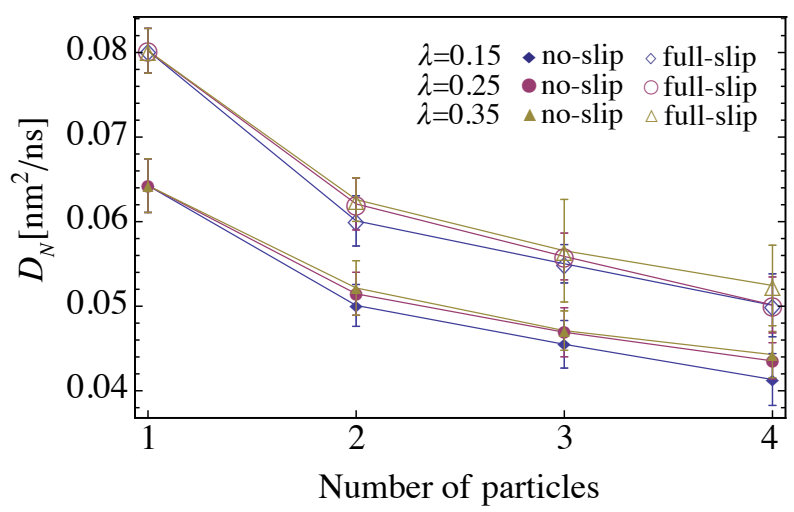

Figure 4: Diffusivities of the colloidal spherical monomers, and, dimers, trimers and tetramers made from spherical monomers of radius $R=2.718 \mathrm{~nm}$ with varying degrees of overlap $\lambda$.

distance between the centers of mass of the constituent monomers. Figure 4 shows the diffusion coefficients for the no-slip and full slip fused nanoclusters with varying degrees of overlap $(\lambda=0.15,0.25$ and 0.35$)$ of the spherical monomers $(R=2.718 \mathrm{~nm})$. A reduction in the size of the fused nanoclusters due to an increase in the degree of overlap $\lambda$ of the monomers reduces both its effective surface area in contact with the fluid and its hydrodynamic radius. Thus, an increase in the degree of overlap $\lambda$ leads to an increase in the diffusivity of the colloidal nanoclusters (Figure 4). Although this increase is appreciable, it only makes for an approximately $4 \%$ change in the diffusivities of the dimers and the trimers, and a $7 \%$ change in the diffusivity of the tetramers. Additionally, a full-slip velocity boundary condition at the fluid-colloid interface reduces the effective drag force exerted by the fluid on the colloid while a no-slip boundary condition serves to enhance it. Thus, on account of the reduced drag force exerted by the fluid in contact with the colloid surface, the full-slip nanoclusters diffuse much faster than the identical no-slip clusters (Figure 4 ).

Table 1: Green-Kubo diffusion coefficients for the diffusive dynamics of the dimers and the trimers (with constituent monomers of radii $R=2.718 \mathrm{~nm}$ ) along their perpendicular and parallel symmetry axes.

\begin{tabular}{|c|c|c|c|c|}
\hline \multirow{2}{*}{} & \multicolumn{2}{|c|}{ dimer $\left(\times 10^{-2} \mathrm{~nm}^{2} / \mathrm{ns}\right)$} & \multicolumn{2}{c|}{ trimer $\left(\times 10^{-2} \mathrm{~nm}^{2} / \mathrm{ns}\right)$} \\
\cline { 2 - 5 } & no-slip & full-slip & no-slip & full-slip \\
\hline$c_{\perp}$ & $4.88 \pm 0.01$ & $6.02 \pm 0.01$ & $4.27 \pm 0.01$ & $5.23 \pm 0.01$ \\
\hline$c_{\|}$ & $5.44 \pm 0.01$ & $6.38 \pm 0.01$ & $4.65 \pm 0.01$ & $5.51 \pm 0.01$ \\
\hline
\end{tabular}


The shape of a colloidal particle influences its diffusivity in different directions along its axes of symmetry. To study this in the diffusivity of a fused nanocluster, we calculated the (anisotropic) Green-Kubo diffusion coefficients ${ }^{48} c_{\perp(\|)}$ for the diffusing dimers and trimers (monomer radii, $R=2.718 \mathrm{~nm}$ ) by resolving their instantaneous velocity $\mathbf{v}(t)$ along directions perpendicular $\left(v_{\perp}\right)$ and parallel $\left(v_{\|}\right)$to a chosen axis of symmetry:

$$
c_{\perp(\|)}=\int_{0}^{\infty}\left\langle v_{\perp(\|)}\left(t+t^{\prime}\right) v_{\perp(\|)}(t)\right\rangle d t^{\prime} .
$$

For the dimer, the parallel component $v_{\|}$was defined to be along the principal axis connecting the centers of mass of the two spherical monomers, and the perpendicular component $v_{\perp}$ perpendicular to it. For the trimer, the parallel component $v_{\|}$was defined to be along an axis connecting the center of mass of the trimer and the center of mass of one of the constituent monomers. The perpendicular axis of the trimer was then defined to be in a direction perpendicular to the plane of the trimer and passing through its center of mass. Table 1 presents the calculated Green-Kubo diffusion coefficients $c_{\perp(\|)}$ for the two fused nanoclusters with no-slip and full-slip boundary conditions. The transport coefficients $c_{\perp}$ and $c_{\|}$for the full-slip boundary condition are larger than those for the no-slip case for both nanoclusters. Also, $c_{\perp}$ is observed to be smaller than $c_{\|}$for both dimers and trimers because increased areas of cross-section in a direction perpendicular to the planes containing the centers of masses of the constituent monomers result in an increased drag force being exerted by the fluid.

Diffusion of macromolecular structures, such as proteins, glycolipids, etc, and their agglomerates plays an important role in the dynamics of biophysical systems. ${ }^{49}$ Also, different experimental conditions during synthesis of colloids and aggregation of colloids in suspensions over time may give rise to colloidal aggregates of various packing fractions, sizes and shapes characterized by a wide range of diffusivities.17 In all such systems, scaling of diffusivity with particle size is important. Figure 5 shows the normalized diffusivities $D_{N} / D_{1}$ 
obtained using FLB for rigid close- and loose-packed colloidal cluster aggregates as a function of the number $N$ of the non-overlapping spherical constituents comprising the aggregates for $N \leq 108$ (see Supporting Information Figure S2 for a schematic of the aggregates and Figure S3 for the distribution of nearest neighbor distances). For comparison, normalized diffusivities of aggregates with $N \leq 147$ are also presented for results obtained using SRD-MD and BD-YRP.17 The normalized diffusivities for the colloidal aggregates obtained from either SRD-MD, BD-YRP or FLB asymptotically follow a power law dependence on the number $N$ of the constituent monomers. Based on a simple volume argument, with an increase in the number $N$ of monomers, the normalized diffusivities of the colloidal aggregates are expected to follow the relation

$$
\frac{D_{N}}{D_{1}}=\frac{R_{H 1}}{R_{H N}} \approx N^{-\alpha}
$$

where $\alpha \rightarrow 1 / 3$ in the continuum limit. Here $R_{H 1}$ and $R_{H N}$ are the hydrodynamic radii of the monomer and the colloidal aggregate, respectively. Results from BD-YRP and FLB simulations of close-packed colloidal aggregates gave scaling exponent values of $\alpha=0.35 \pm$ 0.01 and $0.35 \pm 0.02$, respectively, whereas a value of $0.60 \pm 0.01$ was obtained from the SRDMD simulations. Thus, both BD-YRP and FLB results recover values of the scaling exponent $\alpha$ close to the theoretically expected value of $1 / 3$ obtained in the continuum limit. We note that both in the SRD-MD and BD-YRP simulations the monomers were held together by a Lennard-Jones potential, which allows for dynamical fluctuations in the shapes of the aggregates. 17 The cause of the discrepancy in the exponent between SRD-MD and the other techniques is under investigation. For loose-packed colloidal aggregates, FLB results yielded a scaling exponent of $\alpha=0.37 \pm 0.01$ which is close to the value obtained for the close-packed aggregates. Figure 5 inset further reveals that the diffusivities of the loose-packed aggregates are slightly lower compared to those obtained for the close-packed aggregates on account of an increase in the aggregate size. However, there were no significant differences observed 
between the diffusivities of the close- and loose-packed colloidal cluster aggregates.

Although BD-YRP approaches the scaling exponent of $\alpha=1 / 3$, it makes use of truncated hydrodynamic interactions between colloidal particles due to the use of the analytic YRP diffusion tensor, whereas FLB accounts for full nonlinear hydrodynamic interactions between diffusing particles. Other widely used and successful methods for modeling colloidal diffusion such as the bead or shell models also make use of analytic diffusion tensors for including

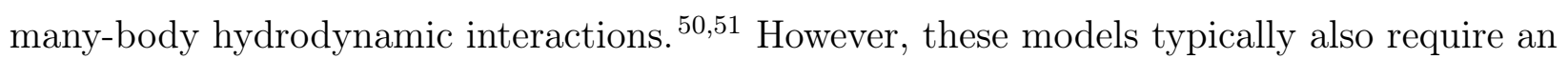
adjustment of the bead radii through a fitting of the experimental rheology data such as intrinsic viscosity, sedimentation rate, etc. to obtain an estimate of the colloidal diffusion coefficient and the hydration layer thickness. ${ }^{52]}$ In contrast, however, FLB simulations do not employ any adjustible parameters for the simulation of colloidal diffusion and hydration layers arise solely as a consequence of the velocity boundary conditions employed at the colloid-fluid interface. Also, among the other mesoscopic methods such as SRD-MD ${ }^{17 / 33}$ and DPD-MD,$\stackrel{33}{3}$ which are based on stochastic coarse-grained Navier-Stokes solvers, FLB is the only method that employs the use of well-defined extended colloidal particles with explicitly implemented microscopic no-slip and full slip velocity boundary conditions at the colloid surface. Further, although results are presented here for the extreme no-slip and full-slip cases, FLB can be easily modified and extended to include continuously varying partial slip at the colloid-fluid interface as well.

To summarize, we have employed FLB to simulate and model the shape and size dependent diffusion of nanoscale colloidal particles, clusters and aggregates, with explicitly implemented microscopic no-slip and full-slip velocity boundary conditions at the colloidfluid interface. Consistent with experimental results for similarly sized colloidal particles, we have obtained effective hydration layer thicknesses associated with colloid surfaces through the use of physically realistic solvent parameters for aqueous solutions. ${ }^{28}[32$ Our results show that the hydration layer contribution to colloidal diffusion at the nanoscale $(1-10 \mathrm{~nm})$ cannot be ignored because at such length-scales the volume of the hydration layer may be a 


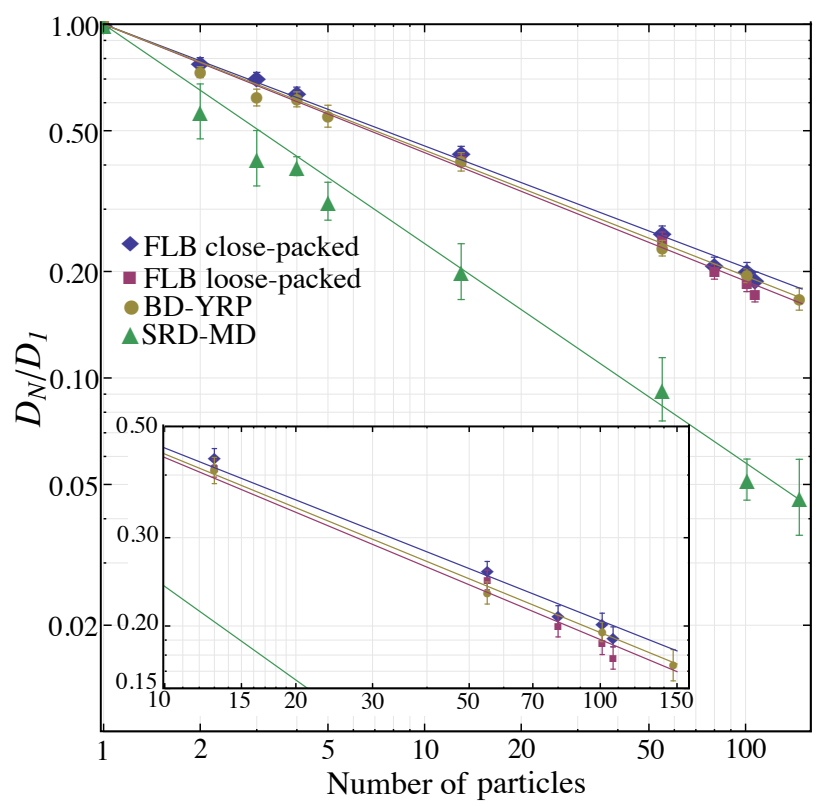

Figure 5: Diffusivities of the colloidal nanoclusters and aggregates (with $N \leq 108$ constituent monomers for the FLB and $N \leq 147$ for the BD-YRP and SRD-MD) normalized by the diffusivity of the single spherical nanocolloid. The lines represent the power law $N^{-\alpha}$ for FLB (rigid close-packed), FLB (rigid loose-packed), BD-YRP and SRD-MD simulations with $\alpha=0.35 \pm 0.02,0.37 \pm 0.01,0.35 \pm 0.01$ and $0.60 \pm 0.01$, respectively. ${ }^{17}$ For the largest FLB clusters the volume fraction of the hydration layer is $\approx 13 \%$.

significant fraction of the colloidal volume. We also observed significant differences in the diffusion of colloids with no-slip and full-slip velocity boundary conditions, wherein full-slip colloids were observed to diffuse faster than no-slip particles of similar size and shape. However, a variation of the degree of overlap of the constituent spherical monomers comprising a colloidal nanocluster did not give rise to any significant diffferences in the diffusivities of the nanoclusters. Green-Kubo diffusion coefficients calculated for colloidal nanoclusters revealed shape dependent anisotropies in diffusivities for both no-slip and full-slip nanocolloids. Finally, FLB simulations were shown to recover the correct scaling exponent for normalized diffusivities of the colloidal nanocluster aggregates in the continuum limit.

In conclusion, our results have revealed striking new features in the shape and size dependent diffusivities of $1-10 \mathrm{~nm}$ colloidal nanoparticles and clusters that manifest themselves only on the nanoscale and had hitherto not been observed for the larger submicron colloids 
in either experimental or theoretical studies.

\section{Acknowledgement}

This work was supported in part by the Academy of Finland through its Centres of Excellence Programme (2012-2017) under Project No. 251748 and Aalto Energy Efficiency Research Programme. We acknowledge the computational resources provided by Aalto Science-IT project, CSC-IT and Structure and Dynamics Group of NIP University of the Philippines. The structural models in Figure 1 were rendered using VMD. 53

\section{Supporting Information Available}

Figure S1. Variation of diffusivities of fused colloidal nanoclusters for the A. no-slip and B. full-slip velocity boundary conditions with length $L$ of the side of a cubic simulation domain; Figure S2. An illustration of the two methods employed for generating randomly assigned coordinates for the spherical monomers in close- and loose-packed colloidal aggregates; Figure S3. Distributions of the nearest-neighbor distances for each spherical monomer in the colloidal cluster aggregates. This material is available free of charge via the Internet at http://pubs.acs.org//.

\section{References}

(1) You, S. M.; Kim, J. H.; Kim, K. H. Effect of nanoparticles on critical heat flux of water in pool boiling heat transfer. Appl. Phys. Lett. 2003, 83, 3374.

(2) Meriläinen, A.; Seppälä, A.; Saari, K.; Seitsonen, J.; Ruokolainen, J.; Puisto, S.; Rostedt, N.; Ala-Nissila, T. Influence of particle size and shape on turbulent heat transfer characteristics and pressure losses in water-based nanofluids. Int. J. Heat Mass Transf. 2013, 61, 439-448. 
(3) Wang, X.-Q.; Mujumdar, A. S. Heat transfer characteristics of nanofluids: a review. Int. J. Therm. Sci. 2007, 46, 1-19.

(4) Jang, S. P.; Choi, S. U. S. Role of Brownian motion in the enhanced thermal conductivity of nanofluids. Appl. Phys. Lett. 2004, 84, 4316.

(5) Soppimath, K. S.; Aminabhavi, T. M.; Kulkarni, A. R.; Rudzinski, W. E. Biodegradable polymeric nanoparticles as drug delivery devices. J. Control. Release 2001, 70, 1-20.

(6) Cho, E. C.; Zhang, Q.; Xia, Y. The effect of sedimentation and diffusion on cellular uptake of gold nanoparticles. Nat. Nanotechnol. 2011, 6, 385-91.

(7) Vogel, N.; Weiss, C. K.; Landfester, K. From soft to hard: the generation of functional and complex colloidal monolayers for nanolithography. Soft Matter 2012, 8, 4044-4061.

(8) Cerbelaud, M.; Videcoq, A.; Abélard, P.; Pagnoux, C.; Rossignol, F.; Ferrando, R. Heteroaggregation between Al2O3 submicrometer particles and $\mathrm{SiO} 2$ nanoparticles: Experiment and simulation. Langmuir 2008, 24, 3001-3008.

(9) Piechowiak, M. A.; Videcoq, A.; Rossignol, F.; Pagnoux, C.; Carrion, C.; Cerbelaud, M.; Ferrando, R. Oppositely Charged Model Ceramic Colloids: Numerical Predictions and Experimental Observations by Confocal Laser Scanning Microscopy. Langmuir 2010, 26, 12540-12547.

(10) Li, F.; Josephson, D. P.; Stein, A. Colloidal assembly: the road from particles to colloidal molecules and crystals. Angew. Chem. Int. Ed. Engl. 2011, 50, 360-88.

(11) Hong, L.; Anthony, S. M.; Granick, S. Rotation in suspension of a rod-shaped colloid. Langmuir 2006, 22, 7128-31.

(12) Hoffmann, M.; Wagner, C. S.; Harnau, L.; Wittemann, A. 3D Brownian diffusion of submicron-sized particle clusters. ACS Nano 2009, 3, 3326-34. 
(13) Happel, J.; Brenner, H. Low Reynolds number hydrodynamics: with special applications to particulate media; Springer, 1983; Vol. 1.

(14) Snook, I.; O’Malley, B.; McPhie, M.; Daivis, P. The approach to the Brownian limit in particulate dispersions. J. Mol. Liq. 2003, 103-104, 405-421.

(15) Shin, H. K.; Kim, C.; Talkner, P.; Lee, E. K. Brownian motion from molecular dynamics. Chem. Phys. 2010, 375, 316-326.

(16) Li, Z. Critical particle size where the Stokes-Einstein relation breaks down. Phys. Rev. E 2009, 80, 061204.

(17) Tomilov, A.; Videcoq, A.; Cerbelaud, M.; Piechowiak, M. A.; Chartier, T.; AlaNissila, T.; Bochicchio, D.; Ferrando, R. Aggregation in colloidal suspensions: evaluation of the role of hydrodynamic interactions by means of numerical simulations. $J$. Phys. Chem. B 2013, 117, 14509-17.

(18) Zhu, Y.; Granick, S. Limits of the Hydrodynamic No-Slip Boundary Condition. Phys. Rev. Lett. 2002, 88, 106102.

(19) Richardson, S. On the no-slip boundary condition. J. Fluid Mech. 1973, 59, 707-719.

(20) Lauga, E.; Brenner, M.; Stone, H. Microfluidics : The No-Slip Boundary Condition. Springer Handb. Exp. Fluid Mech. 2007, 1219-1240.

(21) Carrasco, B.; García de la Torre, J. Hydrodynamic properties of rigid particles: comparison of different modeling and computational procedures. Biophys. J. 1999, 76, $3044-57$.

(22) Batchelor, G. K. An introduction to fluid dynamics; Cambridge University Press, 2000.

(23) Neto, C.; Evans, D. R.; Bonaccurso, E.; Butt, H.-J.; Craig, V. S. J. Boundary slip in Newtonian liquids: a review of experimental studies. Reports Prog. Phys. 2005, 68, $2859-2897$. 
(24) Jiang, Y.; Blanchard, G. J. Rotational Diffusion Dynamics of Perylene in n-Alkanes. Observation of a Solvent Length-Dependent Change of Boundary Condition. J. Phys. Chem. 1994, 98, 6436-6440.

(25) Hay, C. E.; Marken, F.; Blanchard, G. J. Solvent-dependent changes in molecular reorientation dynamics: the role of solvent-solvent interactions. J. Phys. Chem. A 2010, $114,4957-62$.

(26) Mannekutla, J. R.; Inamdar, S. R.; Mulimani, B. G.; Savadatti, M. I. Rotational diffusion of coumarins: a dielectric friction study. J. Fluoresc. 2010, 20, 797-808.

(27) Dutt, G. B.; Sachdeva, A. Temperature-dependent rotational relaxation in a viscous alkane: Interplay of shape factor and boundary condition on molecular rotation. $J$. Chem. Phys. 2003, 118, 8307.

(28) Ebbinghaus, S.; Kim, S. J.; Heyden, M.; Yu, X.; Heugen, U.; Gruebele, M.; Leitner, D. M.; Havenith, M. An extended dynamical hydration shell around proteins. Proc. Natl. Acad. Sci. U. S. A. 2007, 104, 20749-52.

(29) Møller, U.; Cooke, D. G.; Tanaka, K.; Jepsen, P. U. Terahertz reflection spectroscopy of Debye relaxation in polar liquids [Invited]. J. Opt. Soc. Am. B 2009, 26, A113-A125.

(30) Duponchel, L.; Laurette, S.; Hatirnaz, B.; Treizebre, a.; Affouard, F.; Bocquet, B. Terahertz microfluidic sensor for in situ exploration of hydration shell of molecules. Chemom. Intell. Lab. Syst. 2013, 123, 28-35.

(31) Heugen, U.; Schwaab, G.; Bründermann, E.; Heyden, M.; Yu, X.; Leitner, D. M.; Havenith, M. Solute-induced retardation of water dynamics probed directly by terahertz spectroscopy. Proc. Natl. Acad. Sci. U. S. A. 2006, 103, 12301-6.

(32) Salorinne, K.; Lahtinen, T.; Koivisto, J.; Kalenius, E.; Nissinen, M.; Pettersson, M.; 
Häkkinen, H. Nondestructive Size Determination of Thiol-Stabilized Gold Nanoclusters in Solution by Diffusion Ordered NMR Spectroscopy. Anal. Chem. 2013, 85, 3489-3492.

(33) Padding, J.; Louis, A. Hydrodynamic interactions and Brownian forces in colloidal suspensions: Coarse-graining over time and length scales. Phys. Rev. E 2006, 74, 031402 .

(34) Jardat, M.; Bernard, O.; Turq, P.; Kneller, G. R. Transport coefficients of electrolyte solutions from Smart Brownian dynamics simulations. J. Chem. Phys 1999, 110, 79937999.

(35) Malevanets, A.; Kapral, R. Mesoscopic model for solvent dynamics. J. Chem. Phys. 1999, 110, 8605.

(36) Hoogerbrugge, P.; Koelman, J. Simulating microscopic hydrodynamic phenomena with dissipative particle dynamics. Europhys. Lett. 1992, 19, 155.

(37) Kikuchi, N.; Pooley, C. M.; Ryder, J. F.; Yeomans, J. M. Transport coefficients of a mesoscopic fluid dynamics model. J. Chem. Phys. 2003, 119, 6388.

(38) Ollila, S. T. T.; Denniston, C.; Karttunen, M.; Ala-Nissila, T. Fluctuating latticeBoltzmann model for complex fluids. J. Chem. Phys. 2011, 134, 064902.

(39) Ollila, S. T. T.; Smith, C.; Ala-Nissila, T.; Denniston, C. The Hydrodynamic Radius of Particles in the Hybrid Lattice Boltzmann-Molecular Dynamics Method. Multiscale Model. Simul. 2013, 11, 213-243.

(40) Tomilov, A.; Videcoq, A.; Chartier, T.; Ala-Nissila, T.; Vattulainen, I. Tracer diffusion in colloidal suspensions under dilute and crowded conditions with hydrodynamic interactions. J. Chem. Phys 2012, 137.

(41) Mackay, F.; Ollila, S.; Denniston, C. Hydrodynamic forces implemented into LAMMPS through a lattice-Boltzmann fluid. Comput. Phys. Commun. 2013, 184, 2021-2031. 
(42) Plimpton, S. Fast parallel algorithms for short-range molecular dynamics. J. Comput. Phys. 1995, 117, 1-19.

(43) Punkkinen, O.; Falck, E.; Vattulainen, I.; Ala-Nissila, T. Dynamics and scaling of polymers in a dilute solution: analytical treatment in two and higher dimensions. $J$. Chem. Phys. 2005, 122, 094904.

(44) Yeh, I.-C.; Hummer, G. System-Size Dependence of Diffusion Coefficients and Viscosities from Molecular Dynamics Simulations with Periodic Boundary Conditions. J. Phys. Chem. B 2004, 108, 15873-15879.

(45) Lobaskin, V.; Dünweg, B. A new model for simulating colloidal dynamics. New J. Phys. $2004,6,54-54$.

(46) Heyes, D. M.; Cass, M. J.; Powles, J. G.; Evans, W. A. B. Self-diffusion coefficient of the hard-sphere fluid: system size dependence and empirical correlations. J. Phys. Chem. B 2007, 111, 1455-64.

(47) Halle, B.; Davidovic, M. Biomolecular hydration: from water dynamics to hydrodynamics. Proc. Natl. Acad. Sci. U. S. A. 2003, 100, 12135-40.

(48) Evans, D. J.; Morriss, G. P. Statistical mechanics of nonequilibrium liquids; Anu E Press, 2007.

(49) Schmit, J. D.; Ghosh, K.; Dill, K. What drives amyloid molecules to assemble into oligomers and fibrils? Biophys. J. 2011, 100, 450-458.

(50) Garcia de la Torre, J.; Navarro, S.; Lopez Martinez, M. C.; Diaz, F. G.; Lopez Cascales, J. J. HYDRO: a computer program for the prediction of hydrodynamic properties of macromolecules. Biophys. J. 1994, 67, 530-1. 
(51) García de la Torre, J.; del Rio Echenique, G.; Ortega, A. Improved calculation of rotational diffusion and intrinsic viscosity of bead models for macromolecules and nanoparticles. The Journal of Physical Chemistry B 2007, 111, 955-961.

(52) Garcia de la Torre, J. Hydration from hydrodynamics. General considerations and applications of bead modelling to globular proteins. Biophys. Chem. 2001, 93, 159-70.

(53) Humphrey, W.; Dalke, A.; Schulten, K. VMD: visual molecular dynamics. Journal of molecular graphics 1996, 14, 33-38. 
Graphical TOC Entry

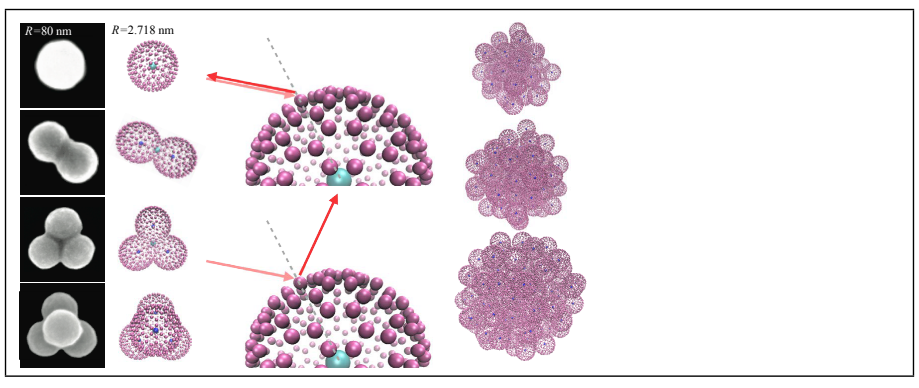

